POS $\quad$ PROCEEDINGS

\title{
Mapping LMXBs with reprocessed X-ray bursts
}

\section{Teo Muñoz-Darias*}

INAF Osservatorio Astronomico di Brera

E-mail: tmd@brera.inaf.it

\section{G. Martínez-Pais \& J. Casares}

Instituto de Astrofísica de Canarias, 38200 La Laguna, Tenerife, Spain

\section{K. O’Brien}

Department of Physics University of California Santa Barbara, CA 93106 USA

\section{R. Cornelisse}

Instituto de Astrofísica de Canarias, 38200 La Laguna, Tenerife, Spain

\section{S. Dhillon}

Dept. of Physics \& Astronomy, Univ. of Sheffield, Sheffield S3 7RH, UK

\section{T. R. Marsh \& D. Steeghs}

Dept. of Physics, Univ. of Warwick, Coventry CV4 7AL, UK

\section{P. A. Charles \& M. Still}

South Africa astronomical observatory, PO Box 9, observatory, South Africa

\begin{abstract}
We present simultaneous high time resolution (1-10 Hz) X-ray and optical observations of the LMXBs Sco X-1 and 4U 1636-536. For Sco X-1 we find that the Bowen/HeII emission lags the $\mathrm{X}$-ray light-curves with a light travel time of $\sim 11-16 \mathrm{~s}$ which is consistent with reprocessing in the donor star. We also present the detection of three correlated X-ray/optical bursts in 4U 1636536. Our results obtained by subtracting the Continuum light-curve from the Bowen/HeII data provide evidence of orbital phase dependent echoes from the companion star. This work shows how bursters are probably the best candidates for applying this technique.
\end{abstract}

High Time Resolution Astrophysics (HTRA) IV - The Era of Extremely Large Telescopes May 5 - 7, 2010

Agios Nikolaos, Crete Greece

\footnotetext{
* Speaker.
} 


\section{Introduction}

Low mass X-ray binaries (LMXBs) are interacting binaries containing a low mass donor star transferring matter onto a neutron star (NS) or black hole. Mass accretion takes place through an accretion disc, and with temperatures approaching $\sim 10^{8} \mathrm{~K}$, such systems are strong X-ray sources. The mass transfer rate supplied by the donor star, $\dot{M}_{2}$, is driven by the binary/donor evolution and for $\dot{M}_{2}>\dot{M}_{\text {crit }} \sim 10^{-9} M_{\odot} y r^{-1}$ results in persistently bright X-ray sources. In these binaries optical emission is dominated by reprocessing of the powerful X-ray luminosity in the gas around the compact object which usually swamp the spectroscopic features of the weak companions stars. In this scenario, dynamical studies have classically been restricted to the analysis of X-ray transients during quiescence, where the intrinsic luminosity of the donor dominates the light spectrum of the binary.

Fortunately, this situation has recently changed thanks to the discovery of the narrow emission components arising from the donor star in the prototypical persistent LMXB Sco X-1 [17]. High resolution spectroscopy revealed many narrow high-excitation emission lines, the most prominent associated with HeII $\lambda 4686$ and NIII $\lambda \lambda 4634-41$ / CIII $\lambda \lambda 4647-50$ at the core of the broad Bowen blend. In particular, the NIII lines are powered by fluorescence resonance through cascade recombination which initially requires EUV seed photons of HeII Ly $\alpha$. These very narrow (FWHM $\leq 50 \mathrm{~km} \mathrm{~s}^{-1}$ ) components move in antiphase with respect to the wings of HeII $\lambda 4686$, which approximately trace the motion of the compact star. Both properties (narrowness and phase offset) imply that these components originate in the irradiated face of the donor star. We repeated this experiment with success in other ten neutron star systems (see e.g. [14]) and in the black hole binary GX339-4 ([9], [13]).

\subsection{Echo-Tomography}

Echo-tomography is an indirect imaging technique which uses time delays between X-ray and UV/optical light-curves as a function of orbital phase in order to map the reprocessing sites in a binary [16]. The optical light curve can be simulated by the convolution of the (source) X-ray light curve with a transfer function which encodes information about the geometry and visibility of the reprocessing regions. The transfer function (TF) quantifies the binary response to the irradiated flux as a function of the lag time and it is expected to have two main components: the accretion disc and the donor star. The latter is strongly dependent on the inclination angle, binary separation and mass ratio and, therefore, can be used to set tight constraints on these fundamental parameters. Successful echo-tomography experiments have been performed on several X-ray active LMXBs using X-ray and broad-band UV/optical light-curves. The results indicate that the reprocessed flux is dominated by the large contribution of the accretion disc (e.g. [8],[16]) which dilutes the reprocessed signal arising from the companion. Only [10] and [4] have reported the detection of reprocessed pulsations in the X-ray binaries 4U 1626-67 and Her X-1 respectively. More recently, [7] have reported some evidence for a reprocessed signal from the companion through the detection of orbital phase dependent echoes in EXO 0748-676. 


\section{Bowen blend echo-tomography of LMXBs}

Based on our previous works in which we detected reprocessed signal from the donor star in many LMXBs, we developped the idea of Bowen blend echo-tomography with the aim of apply this technique to those systems. To do this, we manufactured two narrow (FWHM $=100 \AA$ ) interference filters centered at the Bowen and HeII lines $\left(\lambda_{\text {eff }}=4660 \AA\right)$ and a featureless region at $\lambda_{\text {eff }}=6000 \AA$. These filters were built to be used with ULTRACAM, a triple-beam CCD camera which uses two dichroics to split the light into 3 spectral ranges: Blue $(<\lambda 3900)$, Green $(\lambda \lambda 3900-5400)$ and Red $(>\lambda 5400)$. It uses frame transfer $1024 \times 1024$ E2V CCDs which are continuously read out, and are capable of time resolution down to 2 milliseconds by reading only small selected windows (see [5] for details). The combination of ULTRACAM and the narrow band filters allows us to obtain continuum-subtracted light-curves of the high excitation lines where the response of the donor's contribution is amplified by suppressing most of the background continuum light, which is associated with the disc. As a first step on this project, we decided to undertake an echo-tomography campaign on the brightest LMXB of all, Sco X-1 which is also the prototypical $Z$-source. Here we also present results of a new echo-tomography campaign on the X-ray burster $4 \mathrm{U}$ 1636-536.

\subsection{Echoes from the companion in Sco X-1}

Simultaneous X-ray and optical data of Sco X-1 were obtained on the nights of 17-19 May 2004. For the X-ray observations we used the Proportional Counter Array (PCA) onboard the Rossi X-ray Timing Explorer (RXTE) satellite. The STANDARD-1 mode, with a time resolution of $0.125 \mathrm{~s}$, was used for the variability analysis and the STANDARD-2 mode data, with a time resolution of 16s, was used for the spectral analysis. Sco X-1 was observed during 15 RXTE windows of 16-31 minutes length spread over the three nights and yielding $20 \mathrm{ks}$ of data. About $80 \%$ of these observations were also covered with simultaneous optical data.

The optical data were obtained with ULTRACAM at the Cassegrain focus of the $4.2 \mathrm{~m}$ William Herschel Telescope (WHT) at La Palma. We used our interference filters in the green (Bowen+HeII) and red (Continuum) arms of the intruments. A standard $\operatorname{SDSS} u(\lambda 3543 \AA$ ) filter was also mounted in the blue channel. The images were reduced using the ULTRACAM pipeline software with bias subtraction and flatfielding. The exposure time was initially set to $0.1 \mathrm{~s}$ but was increased to $0.25 \mathrm{~s}$ depending on weather conditions.

\subsubsection{Data analysis}

In order to look for correlated variability we performed a comprehensive cross-correlation analysis by using the entire data set in blocks from $2 \mathrm{~min}$ to $30 \mathrm{~min}$ (i.e. one RXTE window).To do this, we used a modified version of the ICF (Interpolation Correlation Function) method which is explained in great detail by [6]. Significant ICF peaks were found only on the night of 18 May, when the system stayed at the Flaring Branch and the X-ray flux rose up to $1.3 \times 10^{4}$ counts $\mathrm{s}^{-1}$. During this night the system shows long episodes of flaring activity (fig. 1 upper panel) and correlated variability between the X-ray data and the optical lightcurve was observed (fig. 1 lower panel). We obtain highly significant correlation peaks in the three considered filters by choosing different data blocks during W3 (see fig 1), especially during the second part of this window where strong correlated variability is present. W3 is centered at orbital phase 0.52 , i.e. very close to 

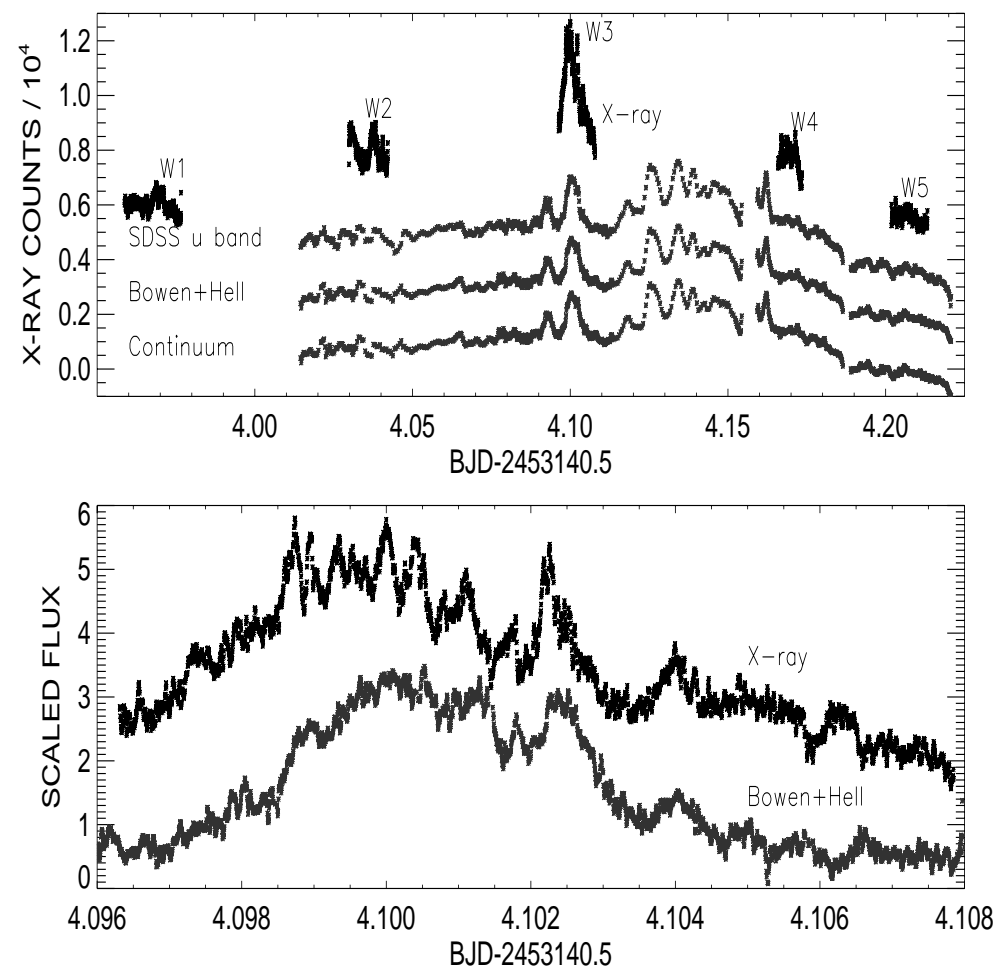

Figure 1: Upper panel: Simultaneous RXTE and ULTRACAM (SDSS $u^{\prime}$, Bowen+HeII and Continuum) observations of Sco X-1/V818 Sco obtained on the night of 18 May 2004. Lower panel: Zoom of W3 showing a 1000s stream of X-ray (above) and Bowen+HeII non continuum subtracted (below) data. The variability appears clearly correlated.

the superior conjunction of the donor star, when the irradiated face presents its largest visibility with respect to us. In many cases we find ICF time-lags of about $11 \mathrm{~s}$ between the X-ray and Bowen+HeII light-curves but we finally selected for a detailed analyis a 3 min block of the W3 where the correlation peaks are particularly significant. Table 1 list the obtained ICF results for the bands considered (i.e. SDSS $u^{\prime}$, Bowen+HeII and Continuum).

With the aim of amplify the signal from the donor we subtracted the Red (Continuum) from the Green(Bowen+HeII) channels. However, the Continuum is also strongly correlated with the Xray emission, and hence, the subtraction process reduce the correlation level of the signal and add noise. In order to determine the optimal amount of continuum to be subtracted to amplify efficiently the signal from the companion, we computed several ICFs after subtracting a fraction $c f$ (with $c f$ in the range 0-1) of the red continuum from the Bowen+HeII light curve. We find that the ICF peak of the continuum subtracted lightcurve (Bowen+HeII CS) smoothly shifts to longer delays for larger $c f$ values, as expected if more disc contribution is subtracted (see upper panel in fig 2). We obtain a delay in the range $14.3-16.3 \mathrm{~s}$ for $c f=0.6-0.8$, when $\sim 60 \%$ of the continuum light is subtracted. For higher $c f$ values the ICF are nosiers and the peaks are not significant.

In a second step in the data anlysis we fitted the ULTRACAM light-curves with the results 
Table 1: TF and ICF analysis for W3

\begin{tabular}{c|ccc}
\hline & $\mathrm{ICF} \pm 1 \sigma(\mathrm{s})$ & $\tau_{0} \pm 1 \sigma(\mathrm{s})$ & $\Delta \tau_{0} \pm 1 \sigma(\mathrm{s})$ \\
\hline Bowen+HeII CS & $14.3-16.3$ & $13.5 \pm 3.0$ & $8.5 \pm 3.5$ \\
Bowen+HeII & $11.8 \pm 0.1$ & $10.75 \pm 1.25$ & $6.25 \pm 1.5$ \\
SDSS $u^{\prime}$ & $9.5 \pm 0.7$ & $9.0 \pm 1.5$ & $5.5 \pm 2.0$ \\
Continuum & $9.0 \pm 0.7$ & $8.5 \pm 1.0$ & $5.5 \pm 1.5$ \\
\hline
\end{tabular}
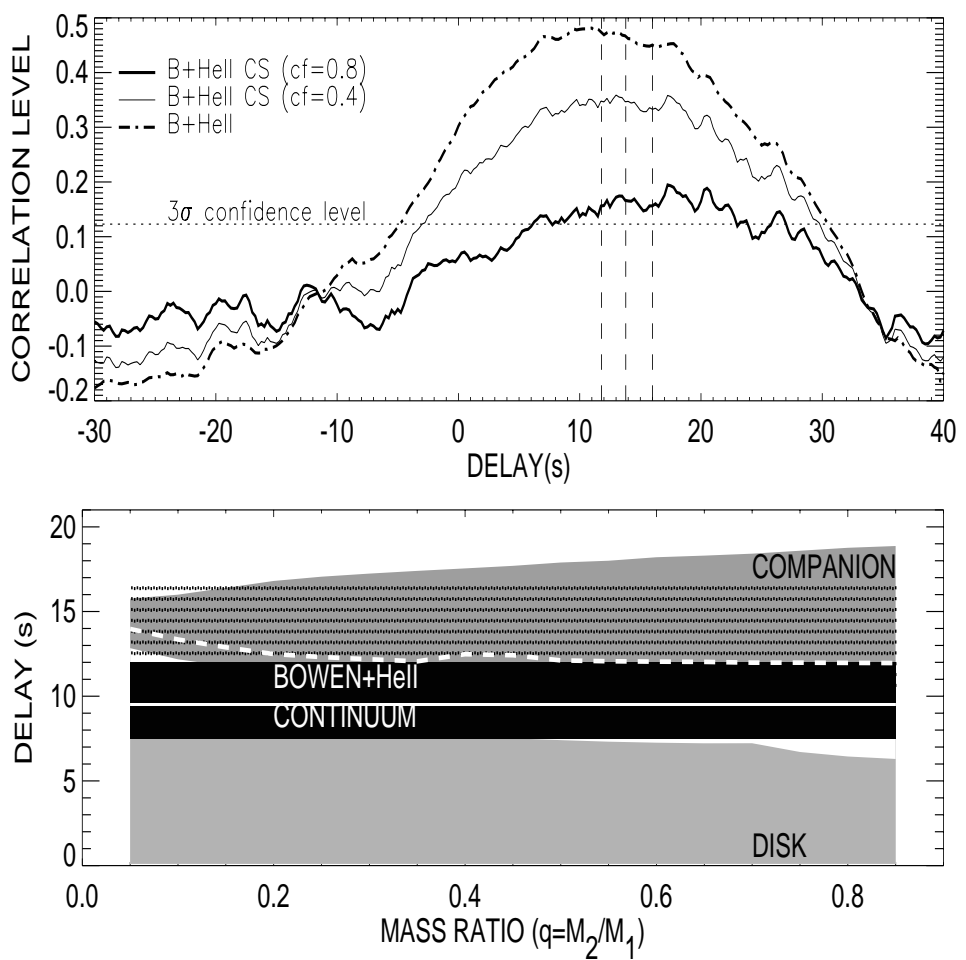

Figure 2: Upper panel: ICF for the selected 3min block of W3. The ICFs have been computed using the Xray data and a Bowen+HeII light curve obtained by subtracting from the green channel the Continuum data scaled by a factor $c f=0$ (i. e. no subtraction), 0.4 and 0.8 . The dotted line shows the $3 \sigma$ confidence level and the dashed vertical lines time-lags of $11.8 \pm 0.1 \mathrm{~s}, 13.3 \pm 0.1 \mathrm{~s}$ and $16.0 \pm 0.3 \mathrm{~s}$ respectively. Lower panel: Expected range of delays for both the companion and the accretion disc considering $i=50^{\circ}, M_{1}=1.4 M_{\odot}$ and the orbital parameters of Sco X-1. The black region shows the $1 \sigma$ delays obtained for the Bowen+HeII, and Continuum data. The shadowed region represents the range of delays obtained by subtracting the Continuum from the Bowen+HeII light-curve. The white dashed line is the highest probability delay for the companion according to [11] and using $\alpha=6^{\circ}$. 
Table 2: Correlated X-ray/optical Type I burst in 4U 1636-536

\begin{tabular}{c|ccccc}
\hline BURST & TIME (MJD) & $\varphi_{\text {orbital }}$ & ICF- $u^{\prime}$ band(s) & ICF-Bowen+HeII(s) & ICF-Continuum(s) \\
\hline Burst-1 & 54271.04381 & 0.22 & $1.45 \pm 0.02$ & $1.73 \pm 0.05$ & $1.73 \pm 0.05$ \\
Burst-2 & 54272.09179 & 0.85 & $1.75 \pm 0.08$ & $1.93 \pm 0.14$ & $2.17 \pm 0.08$ \\
Burst-3 & 54272.20536 & 0.57 & $2.51 \pm 0.06$ & $2.93 \pm 0.06$ & $2.33 \pm 0.05$ \\
\hline
\end{tabular}

of convolving the RXTE data with a set of synthetic transfer functions. For simplicity we have used Gaussian TFs with two free parameters which represent the mean delay ( $\bar{\tau})$ and the standard deviation of the delay $(\Delta \tau)$ :

$$
T F(\tau)=A e^{\frac{1}{2}\left(\frac{\tau-\tau_{0}}{\Delta \tau}\right)^{2}}
$$

where $\mathrm{A}$ is a normalization constant. We have applied this technique to all data sets and find $\bar{y}$ values completely consistent with those obtained in the cross-correlation analysis. A summary of the results is presented in Table 1 .

\subsubsection{Discussion}

We have found positive delays between the X-ray and the three optical bands which we have considered. These time lags are obtained when Sco X-1 stayed at the Flaring Branch and shows high frequency variability. Fig. 2 (lower panel) shows the range of expected time-delays for both the companion and the accretion disc as a function of the mass ratio $\left(q=M_{2} / M_{1}\right)$. The timelags of $\sim 10-11$ s obtained for the Bowen+HeII data during W3 lies at the lower edge of the companion region, and shows that part of this emission must arise from reprocessing on the donor star. For the continuum subtracted data the TF method yields a time-lag of $13.5 \pm 3.0$ s which is perfectly accommodated in the companion region (fig. 2, lower panel). However, the ICF method gives a narrower time-lag range $(14.3-16.3 \mathrm{~s})$. This latter time-lag is larger than expected for the maximum response of the companion and suggests that either the mass of the NS or the orbital inclination are higher than the assumed values. On the other hand the Continuum data shows delays consitent with reprocessing in the accretion disc whereas the $u$ band present larger delays. This is probably due to the presence of many high excitation lines arising from the companion in the spectral range covered by this filter. We note that correlated variability is also found during W5 (fig. 1) when the system shows less activity. In this case we find delays consistent with reprocessing in an accretion disc with a radial temperature profile. (see [12] for details)

\subsection{Phase-dependent echoes from the companion star in $4 \mathrm{U}$ 1636-536/V801 Ara}

Encouraged by the successful echo-tomography work on Sco X-1 we decided to undertake a new campaign on the LMXBs 4 U 1636-536 where [3] detected reprocessed emission from the companion. This system is also a well-known X-ray burster which offers the opotunity of exploiting burst events in order to detect reprocessed signals at different orbital phases. We observed 4 U 1636-536 on 19-20 June 2007 with ULTRACAM attached to the Very Large Telescope (VLT) at Cerro Paranal (Chile). We used the same instrument set up than in the Sco X-1 campaign with 

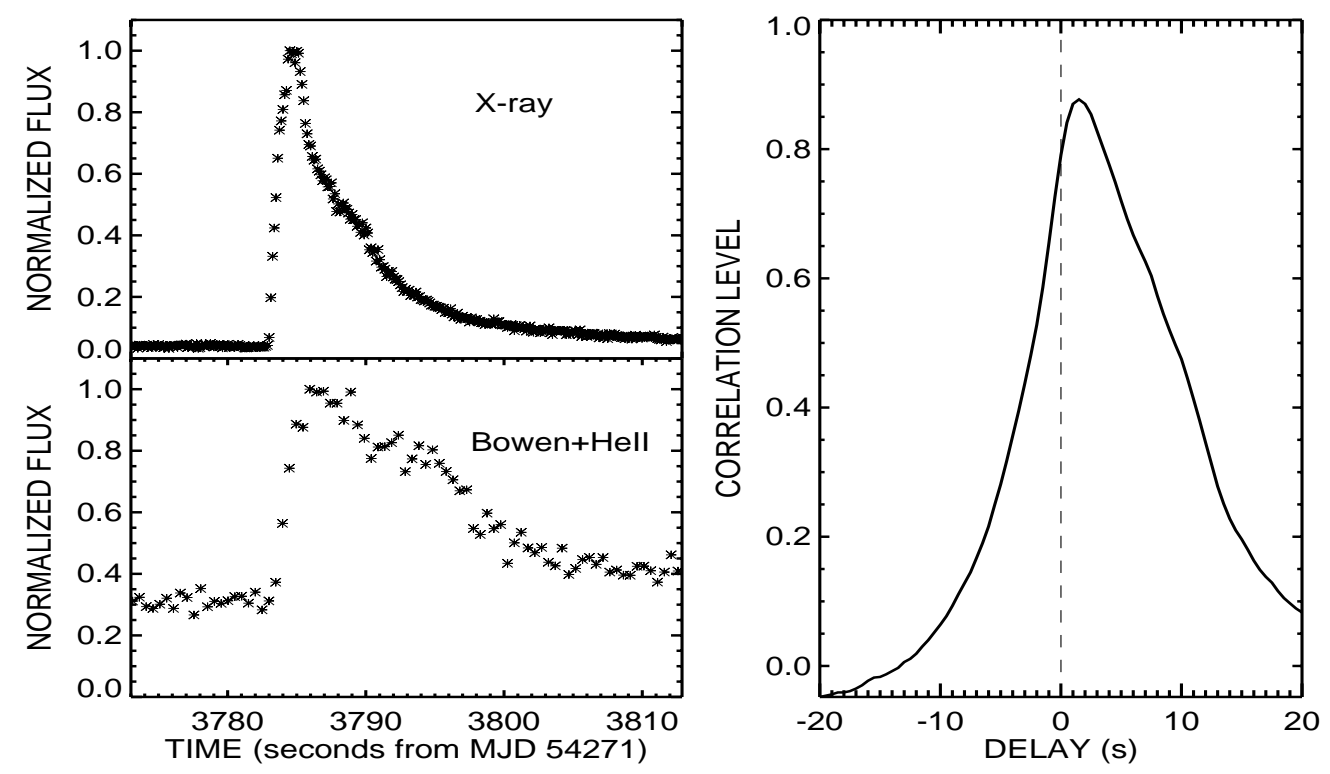

Figure 3: Left panel:40s streams of X-ray (above) and Bowen+HeII (below) data around Burst-1. The smearing in the shape of the optical event is cleary seen in the figure. Righ panel: ICF obtained through the left panel lightcurves. A clear peak centered at $1.73 \pm 0.05$ is found.

a time resolution of $0.5 \mathrm{~s}$, although it was increased up to $1 \mathrm{~s}$ during the 2 nd night when thin clouds appeared. We also obtained simultaneous RXTE data covering the ground based campaing. Here we present a preliminary ULTRACAM-pipeline data reduction together with the STANDARD-1 RXTE data set at $T_{\text {exp }}=0.125 \mathrm{~s}$.

During the 2 nights that we were observing three simultaneous X-ray/optical Burst were detected (see fig. 3 left panel). Table 2 lists the times of the bursts and the orbital phase at which the events occured following the ephemerides gave in [3].

ICF functions were computed in the same way than in the Sco X-1 work by selecting 200s blocks of data around each bursts. We find in all the cases very clear ICF peaks centered at positives delays (i.e optical event occurs after X-ray event). Figure 3 shows the X-ray and Bowen+HeII lightcurves during Burst-1 together with the resulting ICF function (right panel) and in table 2 we report the obtained time lags for all the bands considered. We measure longer delays during Burst-3, at $\varphi_{o r b} \sim 0.57$, when the visibily of the companion is higher. Furthermore, in light with the Sco X-1 study, the delays obtained in the Bowen+HeII filter are longer than those seen in the other bands. In order to amplify the signal from the donor we subtracted the Continuum emission scaled by a continuum factor $(c f)$ from the Bowen+HeII lightcurve. Figure 4 shows the obtained delays for each burst vs $c f$. We also mark (grey regions) the expected range of delays for the companion (following [11]) by considering an orientative set of orbital paramenters of 4U 1636-536 and the orbital phase at which the events were detected. In the three cases the obtained time-lags are consistent with reprocessing on the companion star. We have also fitted the obtained delays by using a simple circular orbit model. The best fit is obtained for an orbital inclination of 45 degrees, consistent with the values $\left(40-60^{\circ}\right)$ favoured by [3]. 


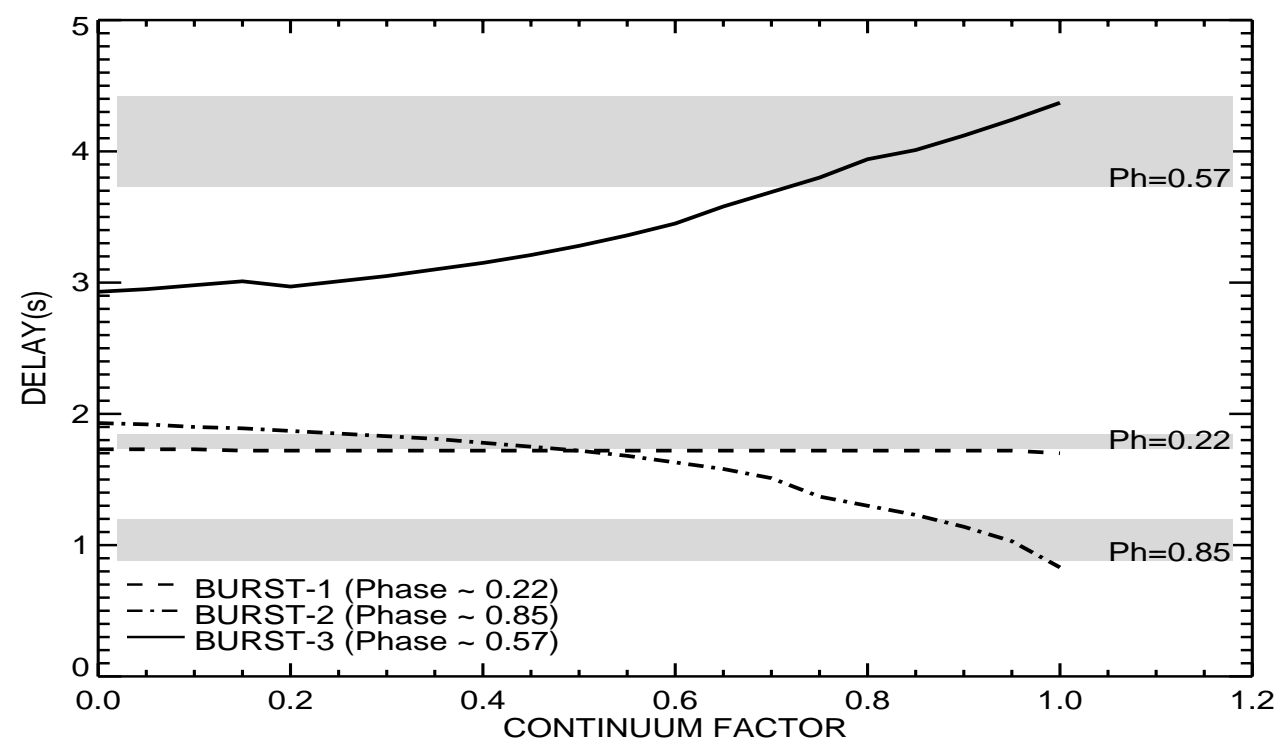

Figure 4: Time-lag vs Continuum factor for the three burst detected. The grey regions show the expected range of delays for the companion assuming $q=0.265$ ([3]), $M_{N S}=1.4 M_{\odot}$, disc flaring angle $(\alpha)=6^{\circ}$, orbital inclination in the range $40-60^{\circ}$ and the orbital phase corresponding to each burst.

Finally we want to note that a new observing campaign on another X-ray burster, EXO 0748676, was performed on January 2008. In this system high excitation emission arising from the companion has been also reported by [15]. We detected four correlated bursts with phase-dependent delays (O'Brien et al. in prep.).

\section{CONCLUSIONS}

Based on the discovering of narrow high excitation emission lines arising from the companion star in many LMXBs we are undertaking several Bowen blend echo-tomography experiments by using ULTRANCAN+narrow band filters and RXTE. In our first work on the prototypical LMXB Sco X-1 we report the detection of echoes consistent with reprocessing on the companion star. The correlated variability was found when the system stayed at the Flaring Branch and displayed high and low frequency variability. The campaing in the X-ray burster $4 \mathrm{U}$ 1636-536 yields evidences of orbital-phase dependent echoes from the companion during the three burst which were detected. This latter work is still on progress and we are currently working on refine our results by fitting time-delayed transfer functions to our data. At the same time we are undertaking new projects in order to apply this promising technique to others LMXBs.

\section{References}

[1] Casares J., Charles P.A. \& Kuulkers E. 1998, ApJ, 493, L39-L42.

[2] Casares J., Steeghs D., Hynes R.I., Charles P.A., Cornelisse R. \& O’Brien K. 2004,RMxA, $20,21$.

[3] Casares, J., Cornelisse, R., Steeghs, D., Charles, P. A., Hynes, R. I., O’Brien, K., \& Strohmayer, T. E. 2006, MNRAS, 373, 1235 
[4] Davidsen, A. Margon, B., Middleditch, J. 1975, ApJ, 198, 653

[5] Dhillon, V.S. et al., 2007, MNRAS,378, 825

[6] Gaskell C.M. \& Peterson B.M. 1987, ApJ, 65, 1.

[7] Hynes, R.I., Horne, K., O’Brien, K., Haswell, C.A., Robinson, E. L., King, A. R., Charles, P. A., \& Pearson, K. J., 2006, ApJ, 648, 1156

[8] Hynes R.I., O’Brien K., Horne K., Chen W. \& Haswell C.A. 1998,MNRAS, 299, L37.

[9] Hynes R.I., Steeghs D., Casares J., Charles P.A.\& O’Brien K. 2003, ApJ, 583, L95.

[10] Middleditch, J., Mason, K. O., Nelson, J. E., \& White, N. E. 1981, ApJ, 244, 1001

[11] Muñoz-Darias T., Martínez-Pais I.G. \& Casares J. 2005, in INTERACTING BINARIES: Accretion, Evolution and Outcomes, eds. L.A. Antonelli, L. Burderi, F. D’Antona, et al.

[12] Muñoz-Darias, T., Martínez-Pais, I. G., Casares, J., Dhillon, V. S., Marsh, T. R., Cornelisse, R., Steeghs, D., \& Charles, P. A. 2007, MNRAS, 616

[13] Muñoz-Darias, T., Casares, J., \& Martínez-Pais, 2008, MNRAS, 385, 2205

[14] Muñoz-Darias, T., 2009, PASP, 121, 935

[15] Muñoz-Darias, T., Casares, J., O’Brien, K., Steeghs, D., Martínez-Pais, Cornelisse, R., \& Charles, P. A. 2009, MNRAS, 394, L136

[16] O’Brien K., Horne K., Hynes R.I., Chen W., Haswell C.A. \& Still M.D. 2002, MNRAS, 334, 426.

[17] Steeghs D. \& Casares J. 2002, ApJ, 568, 273-278. 